\title{
Clinical course of untreated thalamic cavernous malformations: hemorrhage risk and neurological outcomes
}

\author{
${ }^{*}$ Kai-Bing Tian, MD, ${ }^{1-4}$ Jing-Jie Zheng, MD, PhD, ${ }^{5}$ Jun-Peng Ma, MD, ${ }^{1-4}$ Shu-Yu Hao, MD, PhD, ${ }^{1-4}$ \\ Liang Wang, MD, PhD, ${ }^{1-4}$ Li-Wei Zhang, MD, PhD, ${ }^{1-4}$ Zhen Wu, MD, PhD, ${ }^{1-4}$ Jun-Ting Zhang, MD, ${ }^{1-4}$ \\ and $\mathrm{Da} \mathrm{Li}, \mathrm{MD}^{1-4}$
}

\begin{abstract}
${ }^{1}$ Department of Neurosurgery, Beijing Tiantan Hospital, Capital Medical University; ${ }^{2}$ China National Clinical Research Center for Neurological Diseases; ${ }^{3}$ Center of Brain Tumor, Beijing Institute for Brain Disorders; ' ${ }^{4}$ Beijing Key Laboratory of Brian Tumor, Beijing; and ${ }^{5}$ Department of Obstetrics and Gynecology, First Affiliated Hospital of Wenzhou Medical University, Wenzhou, People's Republic of China
\end{abstract}

OBJECTIVE The natural history of cerebral cavernous malformations (CMs) has been widely studied, but the clinical course of untreated thalamic CMs is largely unknown. Hemorrhage of these lesions can be devastating. The authors undertook this study to obtain a prospective hemorrhage rate and provide a better understanding of the prognosis of untreated thalamic CMs.

METHODS This longitudinal cohort study included patients with thalamic CMs who were diagnosed between 2000 and 2015. Clinical data were recorded, radiological studies were extensively reviewed, and follow-up evaluations were performed.

RESULTS A total of 121 patients were included in the study (56.2\% female), with a mean follow-up duration of 3.6 years. The overall annual hemorrhage rate (subsequent to the initial presentation) was calculated to be $9.7 \%$ based on the occurrence of 42 hemorrhages over 433.1 patient-years. This rate was highest in patients $(n=87)$ who initially presented with hemorrhage and focal neurological deficits (FNDs) $(14.1 \%)\left(\chi^{2}=15.358, p<0.001\right)$, followed by patients $(n=19)$ with hemorrhage but without FND $(4.5 \%)$ and patients $(n=15)$ without hemorrhage regardless of symptoms (1.2\%). The initial patient presentations of hemorrhage with FND (hazard ratio [HR] 2.767, 95\% Cl 1.336-5.731, $p=$ 0.006 ) and associated developmental venous anomaly (DVA) (HR 2.510,95\% Cl 1.275-4.942, $p=0.008$ ) were identified as independent hemorrhage risk factors. The annual hemorrhage rate was significantly higher in patients with hemorrhagic presentation at diagnosis $(11.7 \%, p=0.004)$ or DVA $(15.7 \%, p=0.002)$. Compared with the modified Rankin Scale (mRS) score at diagnosis (mean 2.2), the final mRS score (mean 2.0) was improved in 37 patients (30.6\%), stable in 59 patients (48.8\%), and worse in 25 patients (20.7\%). Lesion size (odds ratio [OR] per $0.1 \mathrm{~cm}$ increase $3.410,95 \% \mathrm{Cl}$ 1.272-9.146, $p=0.015$ ) and $m R S$ score at diagnosis (OR per 1 point increase $3.548,95 \% \mathrm{Cl} 1.815-6.937, p<0.001$ ) were independent adverse risk factors for poor neurological outcome (mRS score $\geq 2$ ). Patients experiencing hemorrhage after the initial ictus (OR per 1 ictus increase 6.923, 95\% Cl 3.023-15.855, $p<0.001$ ) had a greater chance of worsened neurological status.

CONCLUSIONS This study verified the adverse predictors for hemorrhage and functional outcomes of thalamic CMs and demonstrated an overall annual symptomatic hemorrhage rate of $9.7 \%$ after the initial presentation. These findings and the mode of initial presentation are useful for clinicians and patients when selecting an appropriate treatment, although the tertiary referral bias of the series should be taken into account.

https://thejns.org/doi/abs/10.3171/2016.8.JNS16934

KEY WORDS cavernoma; cavernous malformation; natural history; thalamus; vascular disorders ABBREVIATIONS CCM = cerebral cavernous malformation; $\mathrm{CM}=$ cavernous malformation; $\mathrm{DVA}=$ developmental venous anomaly; FND = focal neurological deficit; $\mathrm{GK}=$
Gamma Knife; $\mathrm{HR}=$ hazard ratio; $\mathrm{mRS}=$ modified Rankin Scale; $\mathrm{OR}=$ odds ratio.

SUBMITTED April 12, 2016. ACCEPTED August 3, 2016.

INCLUDE WHEN CITING Published online November 11, 2016; DOI: 10.3171/2016.8.JNS16934.

* Drs. Tian, Zheng, and Ma contributed equally to this work. 
$\mathrm{T}$ HE incidence of cavernous malformations (CMs) has been reported as ranging from approximately $0.4 \%$ to $0.8 \%$, with the lesions commonly involving the cerebral white matter or subcortex ${ }^{16}$ and only rarely the thalamus. ${ }^{33,42,48,52}$ Because of the presence of vital neurovascular structures within and surrounding the thalamus, hemorrhagic ictus involving these locations can lead to serious neurological deficits and poor quality of life, and surgical excision is challenging. Due to the rarity of thalamic CMs, an optimal management algorithm has remained undefined, and the literature concerning their natural history is limited. As stated by Al-Shahi Salman et al., ${ }^{6}$ the natural history was one of the top research priorities for the study of cerebral cavernous malformations (CCMs). Prior to the largest study of 46 surgical cases, reported by Rangel-Castilla and Spetzler, ${ }^{48}$ clinical course expectations and potential outcomes were largely unknown and often gleaned from small studies or cases reports. An impressive and thorough review had been performed by Gross et al., ${ }^{16}$ including 33 surgically treated cases from 23 studies, but these studies were subject to selection and reporter bias. ${ }^{9}, 10,12,14,16,20,24,25,34-37,40-44,46,47,51$ To further the understanding of thalamic $\mathrm{CMs}$, we extensively reviewed clinical charts and aimed to provide a hemorrhage rate, hemorrhage risk, and prognosis of untreated CMs in a large group of patients who presented to our institution.

\section{Methods}

\section{Participants and Study Design}

Between January 2000 and January 2015, 164 patients with thalamic CMs were referred to the outpatient department of Beijing Tiantan Hospital. The modified Rankin Scale (mRS) was used to evaluate neurological status. ${ }^{\text {? }}$ Patient selection occurred as depicted in Fig. 1. Surgery was recommended in 31 patients (18.9\%) shortly after diagnosis at our institution in cases demonstrating 1) multiple ictus; 2) lesions $\geq 2 \mathrm{~cm}$ in size; 3 ) $\mathrm{mRS}$ score $\geq 2$; 4) hydrocephalus or intraventricular hemorrhage due to CMs; 5) exophytic lesions or lesions abutting the ependyma; 6) progressive growth in lesion size, deterioration of clinical function, or worsening of mRS score. ${ }^{30,32,33}$ Twelve patients $(7.3 \%)$ were excluded from the study due to previous resection $(\mathrm{n}=2)$ or Gamma Knife $(\mathrm{GK})$ treatment (n $=5$ ) prior to being referred to our institution, no consent to follow-up $(\mathrm{n}=4)$, or severe comorbidities (involving the brainstem, peripheral nerves, or other eloquent lobes) that might affect the accuracy of the neurological function evaluation $(n=1)$. All remaining 121 patients were admitted for observation. In 94 of the 121 cases, the patients were registered and entered into the longitudinal cohort study during that hospital admission; in the remaining 27 cases, the patients were not registered into the study during their initial admission, but their clinical data for the period from the initial admission to the time of enrollment were reviewed. In these 27 patients, the mean from the initial admission to study enrollment was 12.8 months (range 0.2-32.5 months, median 10.3 months).

The criteria for conservative management included 1 or more of the following: 1) nonhemorrhagic, asymptomatic, or accidental lesion; 2) small, deep-seated, and inaccessible lesion; 3) a single hemorrhagic ictus plus mild symptoms; 4) functional neurological improvement or a decrease in hematoma size prior to being referred to our institution; and 5) refusal of surgery due to surgical risk. Radiosurgery was not considered at our institution. Individualized management strategies were developed for each patient. Data were collected, and informed consent was obtained from all study participants. The Beijing Tiantan Hospital Research Ethics Committee approved the study.

\section{Clinical and Demographic Data}

During the first clinical consultation for treatment of thalamic CMs in our institution, clinical chart data were recorded, and MRI scans were extensively reviewed in each case. According to the accepted criteria ${ }^{49,53}$ the diagnosis of thalamic CMs was established by 2 independent neuroradiologists. Patient data, such as family history, birth information, prior hemorrhage ictus and treatment, comorbid neurological disease, and symptoms at the time of diagnosis, were reviewed. Based on prior reported methods, data describing thalamic CMs included anatomical location (lesion side and depth), ${ }^{30-32,48}$ lesion size based on T1-weighted MR images, perilesional edema, Zabramski classification,,$^{53}$ the presence or absence of associated developmental venous anomaly (DVA), and the presence or absence of multiple CMs (based on gradient-echo images) (Table 1) (Figs. 2-4).

According to our previously described methods, ${ }^{30}$ hemorrhage was defined as either intralesional or extralesional based on the MRI studies performed at the time of diagnosis. Initial presentation was classified into 3 subgroups: 1) hemorrhage with associated focal neurological deficits (FNDs), 2) hemorrhage without FND (i.e., headache, dizziness, or other symptoms not directly related to the lesions), and 3) no hemorrhage, regardless of symptoms.

A definite hemorrhage was defined as an overt intra- or extralesional hemorrhage based on radiological evidence with or without clinical event (acute or subacute, new onset or worsening of FND, or severe headache) corresponding to the anatomical location of the thalamic CMs. ${ }^{4,5,30} \mathrm{~A}$ probable hemorrhage was defined as a new clinical event with relevant radiological evidence recorded outside of our facility but unavailable for us to review. The probable hemorrhage, clinical events without radiological evidence, and hemorrhages from CMs of other locations were excluded from the calculation of annual hemorrhage rate. Asymptomatic hemorrhage was defined as an overt intraor extralesional hemorrhage with a change in lesion size or signal intensity but without clinical symptomatology. A dark halo surrounding the lesion on the gradient-echo sequence was not considered a hemorrhagic ictus., ${ }^{4,2730}$

\section{Follow-Up}

The inception point of study participation was the date of diagnosis at our institution, and the end point was the date of a censoring event, including the most recent evaluation, death, surgery, or radiotherapy. MRI was recommended at 3,6, and 12 months after diagnosis and then once every 2 years thereafter; the sequences included T1and $\mathrm{T} 2$-weighted imaging and a T2-weighted gradient- 


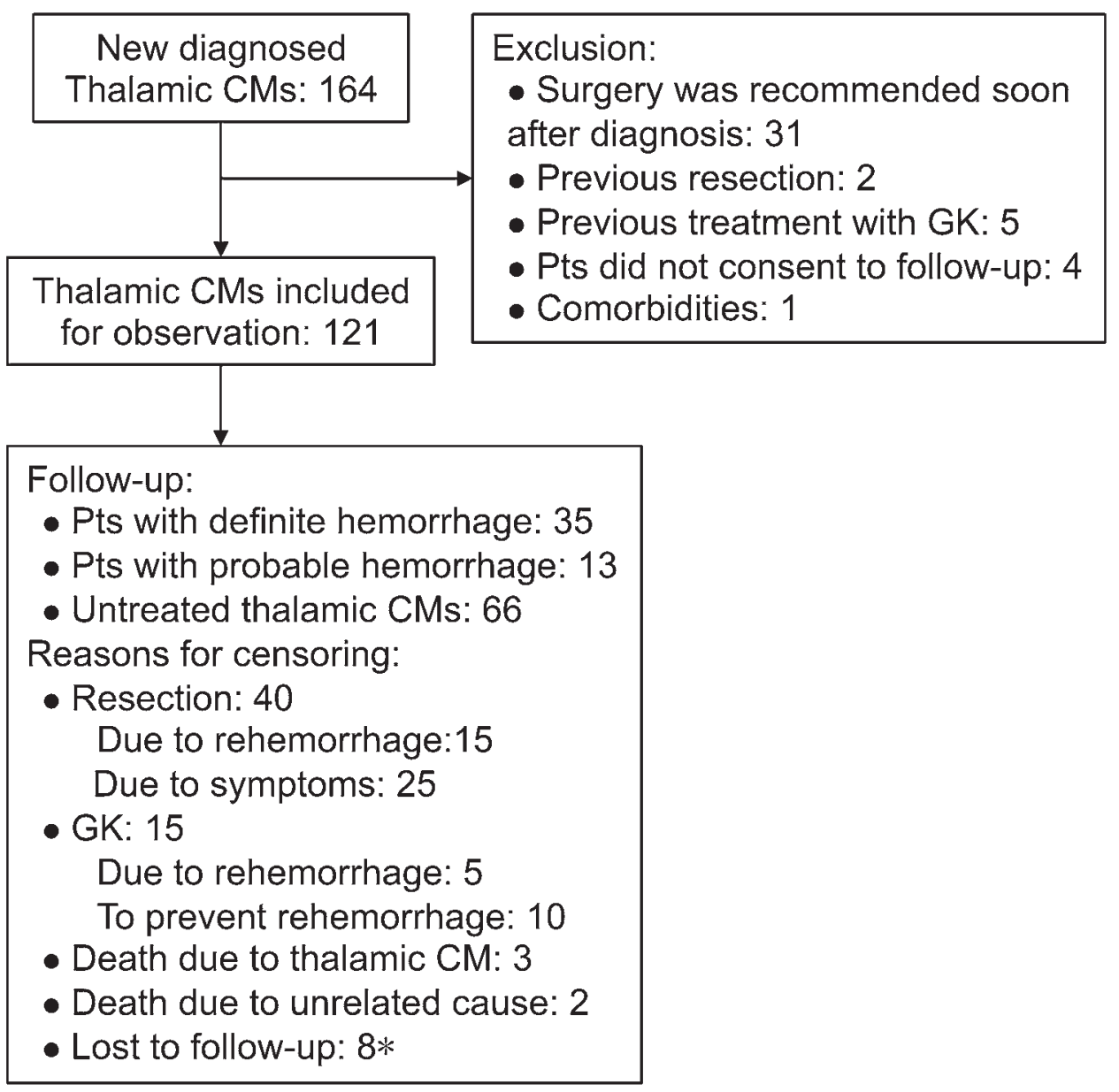

FIG. 1. Flow chart of participants with untreated thalamic CMs. *Eight patients were lost to follow-up before the end of the designated follow-up period, but their most recent follow-up data were obtained and included in analysis. Pts = patients.

echo sequence. Radiological examination was mandatory in cases demonstrating a suspicious hemorrhagic ictus or a clinical event to confirm the presence of hemorrhagic ictus. Prospective follow-up was obtained annually either in the clinic or via telephone, email, or written questionnaire to obtain and review MRI scans., ${ }^{30,31}$ Information was gathered concerning additional treatment since diagnosis, treatment effects, changes in neurological deficits, the most recent $\mathrm{mRS}$ score, and the score for the independent activities of daily living scale.

\section{Statistical Analysis}

The annual hemorrhage rate was calculated as the number of hemorrhages divided by the duration (in years) of follow-up per patient and was further evaluated in patient subgroups of initial presentation and hemorrhage risk (Tables 2 and 3). Analysis of hemorrhage-free survival began on the date of diagnosis and ended on the date of the earliest rehemorrhage or the date of censoring (most recent evaluation, death, CM resection, or radiosurgery), whichever occurred first. ${ }^{5,30,31}$ Hemorrhage risks were verified by multivariate Cox regression analysis, which were further confirmed by the Kaplan-Meier method. The adverse predictors for poor neurological outcomes $(\mathrm{mRS} \geq 2)$ were evaluated by multivariate binary logistic regression analysis. The adverse predictors are listed in Table 3. The posthemorrhagic period was defined as the interval from the date of the last hemorrhage or the diagnosis if there was no hemorrhagic ictus between the initial presentation and the date of censoring. Analyses were performed using IBM SPSS Statistics (version 20.0) with the significance set at $\mathrm{p}<0.05$.

\section{Results}

\section{Patient Characteristics}

A total of 121 patients were included in the study (56.2\% female), and their mean age at diagnosis was 34.1 years (range 2-67 years); their demographic and baseline clinical characteristics are detailed in Table 1. Two patients underwent surgical excision of CMs in other locations (the right occipital lobe in 1 patient and the right temporal lobe in the other) prior to consultation at our institution. A family history of CMs was documented in 3 patients (2.5\%). At diagnosis, 113 patients (93.4\%) suffered a prior hemorrhage ictus, 87 patients (71.9\%) presented with hemorrhage and associated FND, 19 patients $(15.7 \%)$ presented with hemorrhage but without FND, and 
TABLE 1. Demographic and clinical characteristics of 121 patients with thalamic CM stratified by initial presentation

\begin{tabular}{|c|c|c|c|c|c|}
\hline \multirow[b]{2}{*}{ Characteristic } & \multirow[b]{2}{*}{ All Pts $(n=121)$} & \multicolumn{3}{|c|}{ Initial Presentation } & \multirow[b]{2}{*}{$\mathrm{p}$ Value } \\
\hline & & Hemorrhage w/ FND $(n=87)$ & Hemorrhage w/o FND $(n=19)$ & No Hemorrhage $(n=15)$ & \\
\hline Female sex & $68(56.2)$ & $48(55.2)$ & $10(52.6)$ & $10(66.7)$ & $0.669 \dagger$ \\
\hline Mean age, yrs & $34.1 \pm 14.9$ & $34.5 \pm 14.6$ & $26.7 \pm 11.3$ & $41.1 \pm 17.6$ & $0.017^{*} \ddagger$ \\
\hline Pts w/ prior hemorrhage & $113(93.4)$ & $87(100)$ & $18(94.7)$ & $8(53.3)$ & $<0.001^{*} \dagger$ \\
\hline DVA (\%) & $39(32.2)$ & $32(36.8)$ & $4(21.1)$ & $3(20.0)$ & $0.230 \dagger$ \\
\hline Multiple CCMs & $20(16.5)$ & $13(14.9)$ & $2(10.5)$ & $5(33.3)$ & $0.155 \dagger$ \\
\hline Perilesional edema & $66(54.5)$ & $53(60.9)$ & $8(42.1)$ & $5(33.3)$ & $0.069 \dagger$ \\
\hline Zabramski classification & & & & & $<0.001^{*} \dagger$ \\
\hline I & $28(23.1)$ & $23(26.4)$ & $4(21.1)$ & $1(6.7)$ & \\
\hline II & $81(66.9)$ & $64(73.6)$ & $15(78.9)$ & $2(13.3)$ & \\
\hline III/IV & $12(9.9)$ & 0 & 0 & $12(80.0)$ & \\
\hline Mean lesion size, $\mathrm{cm}$ & $2.0 \pm 0.7$ & $2.1 \pm 0.7$ & $2.0 \pm 0.8$ & $1.3 \pm 0.5$ & $<0.001^{*} \ddagger$ \\
\hline Lesion depth & & & & & $0.903 \dagger$ \\
\hline Superficial & $64(52.9)$ & $45(51.7)$ & $11(57.9)$ & $8(53.3)$ & \\
\hline Moderate & $32(26.4)$ & $25(28.7)$ & $4(21.1)$ & $3(20.0)$ & \\
\hline Deep & $25(20.7)$ & $17(19.5)$ & $4(21.1)$ & $4(26.7)$ & \\
\hline \multicolumn{6}{|l|}{ mRS score at diagnosis } \\
\hline Mean & $2.2 \pm 1.1$ & $2.4 \pm 1.0$ & $1.4 \pm 1.0$ & $1.7 \pm 1.1$ & $<0.001^{*} \S$ \\
\hline 0 & 0 & 0 & 1 & 1 & \\
\hline 1 & 36 & 16 & 13 & 7 & \\
\hline 2 & 42 & 34 & 3 & 5 & \\
\hline 3 & 25 & 25 & 0 & 0 & \\
\hline 4 & 15 & 11 & 2 & 2 & \\
\hline 5 & 1 & 1 & 0 & 0 & \\
\hline
\end{tabular}

$\mathrm{Pt}=$ patient.

Values represent numbers of cases (\%) unless otherwise indicated. Mean values are presented with standard deviations. All $p$ values are for comparison of the difference between subgroups.

* Statistically significant $(p<0.05)$.

$\dagger$ Chi-square test.

$\ddagger$ One-way ANOVA.

$\S$ Kruskal-Wallis test.

15 patients (12.4\%) did not present with overt hemorrhage. The mean mRS score was 2.2 (range 0-5) for all patients at diagnosis and 2.4 in patients presenting with hemorrhage and FND.

Based on MRI scans, 62 (51.2\%) and 59 (48.8\%) lesions were located in the left and right thalamus, respectively. Hydrocephalus, DVA, and multiple CCMs were observed in $24(19.8 \%), 39(32.2 \%)$, and $20(16.5 \%)$ patients, respectively. The mean lesion size was $2.0 \mathrm{~cm}$ (range 0.6-3.4 $\mathrm{cm})$. Perilesional edema was noted in 66 lesions $(54.5 \%)$. Zabramski classification Type I, II, and III/IV were determined in 28 (23.1\%), 81 (66.9\%), and 12 (9.9\%) patients, respectively. Lesion depth was characterized as superficial in 64 patients $(52.9 \%)$, moderate in 32 patients $(26.4 \%)$, and deep in 25 patients (20.7\%).

\section{General Data and Follow-Up}

Return visits were recorded for 105 patients (86.8\%), and the remaining 16 patients $(13.2 \%)$ were followed up via telephone, email, and/or written questionnaire alone. Eight patients (6.6\%) were lost to final follow-up evaluation, but their last follow-up data were reviewed and included in the analysis. The mean follow-up duration was 3.6 years (range 0.1-16.6 years, 433.1 patient-years), respectively. MRI reexamination was available and reliable in 103 patients $(85.1 \%)$, and 99 patients $(81.8 \%)$ underwent multiple follow-up MRI studies. Not all patients followed our advice for MRI reexamination at the recommended time points, however, and the MRI scans could be obtained and reviewed only if they had been performed, which depended on patient compliance. Usually, patients who did not return for clinical visit experienced improvement or recovery that reduced their need for medical consultation.

Three patients $(2.5 \%, 2$ females and 1 male) died of thalamic CM hemorrhage. Two patients died of unrelated causes (breast cancer and heart disease). Seven of the 24 patients diagnosed with hydrocephalus were treated with ventriculoperitoneal shunt placement rather than surgical resection of the lesion. Fifteen patients underwent GK therapy (due to rehemorrhage in 5 cases and to prevent rehemorrhage in 10 cases in which the patients had not yet experienced rehemorrhage and were not experiencing symptoms); we did not routinely recommend radiosurgery 

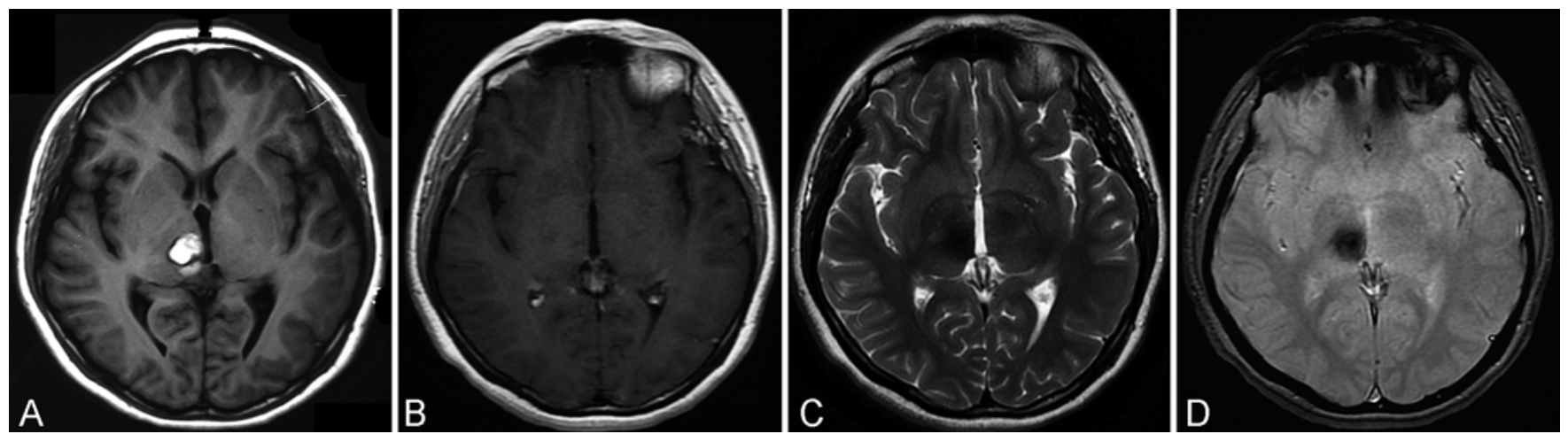

FIG. 2. Case 1. Axial MR images obtained in an untreated patient with a vanishing hematoma. This 17-year-old male patient with an mRS score of 3 at diagnosis suffered a single hemorrhage of a right thalamic CM according to the results of an MRI scan (T1weighted image, A). At the follow-up evaluation 3 months later, the hematoma had disappeared, and the lesion had decreased in size (T1-weighted image, B) with hemosiderin evident on T2-weighted (C) and gradient-echo images (D). After a follow-up duration of 63.9 months, the patient's mRS score had decreased to 0 , and he was symptom free.

in all cases. Forty patients underwent resection (due to rehemorrhage in 15 cases and due to symptoms in 25) as an initial treatment. Two patients underwent repeat GK treatment, and 1 patient underwent resection after GK treatment failure. In the cases in which patients underwent resection, the rehemorrhage or clinical symptoms impelled them to seek treatment. Reevaluation was required for patients suffering rehemorrhage during follow-up, and
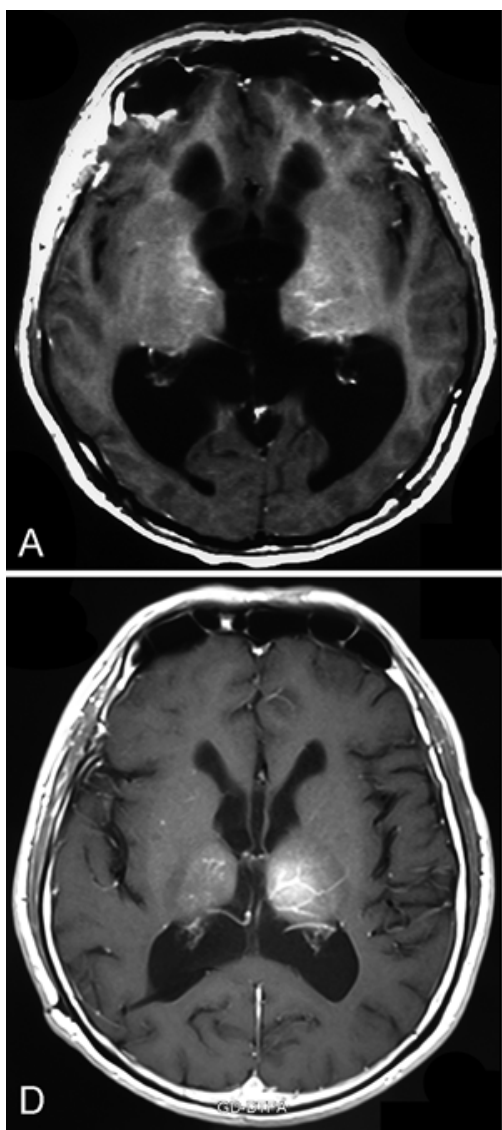
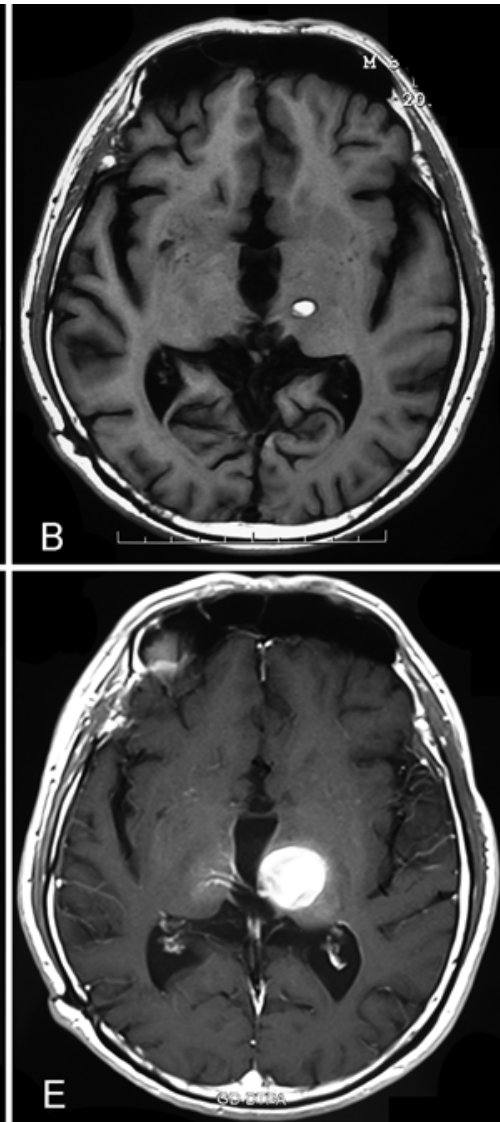
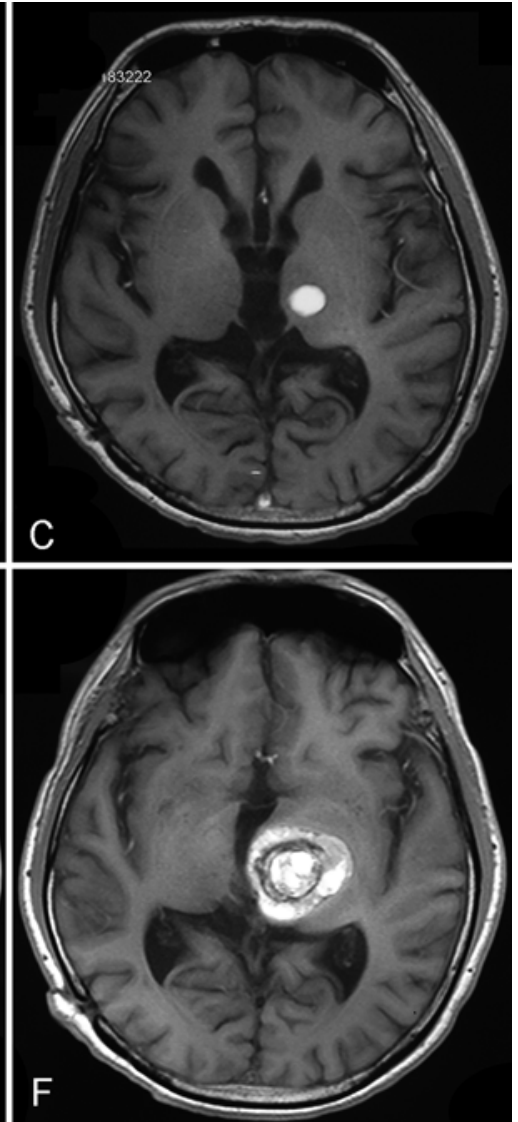

FIG. 3. Case 2. Axial MR images obtained in an untreated patient who suffered multiple hemorrhages. This 37-year-old woman presented with a medical history of hydrocephalus for which she had undergone ventriculoperitoneal shunt placement 7 years previously at another medical facility; evidence of a CM was absent except for an enhancing venous anomaly detected on the T1-weighted contrast-enhanced MRI (A). At the patient's initial visit to our institution, a left thalamic CM was identified on the T1weighted MRI, (B) and the patient had an mRS score of 1 . She subsequently suffered 3 hemorrhages, as shown on T1-weighted contrast-enhanced MRI (first ictus, C; second ictus, D and E; third ictus, F). The associated DVA was remarkable. The patient did not receive any treatment and had an mRS score of 2 at her most recent follow-up visit. 

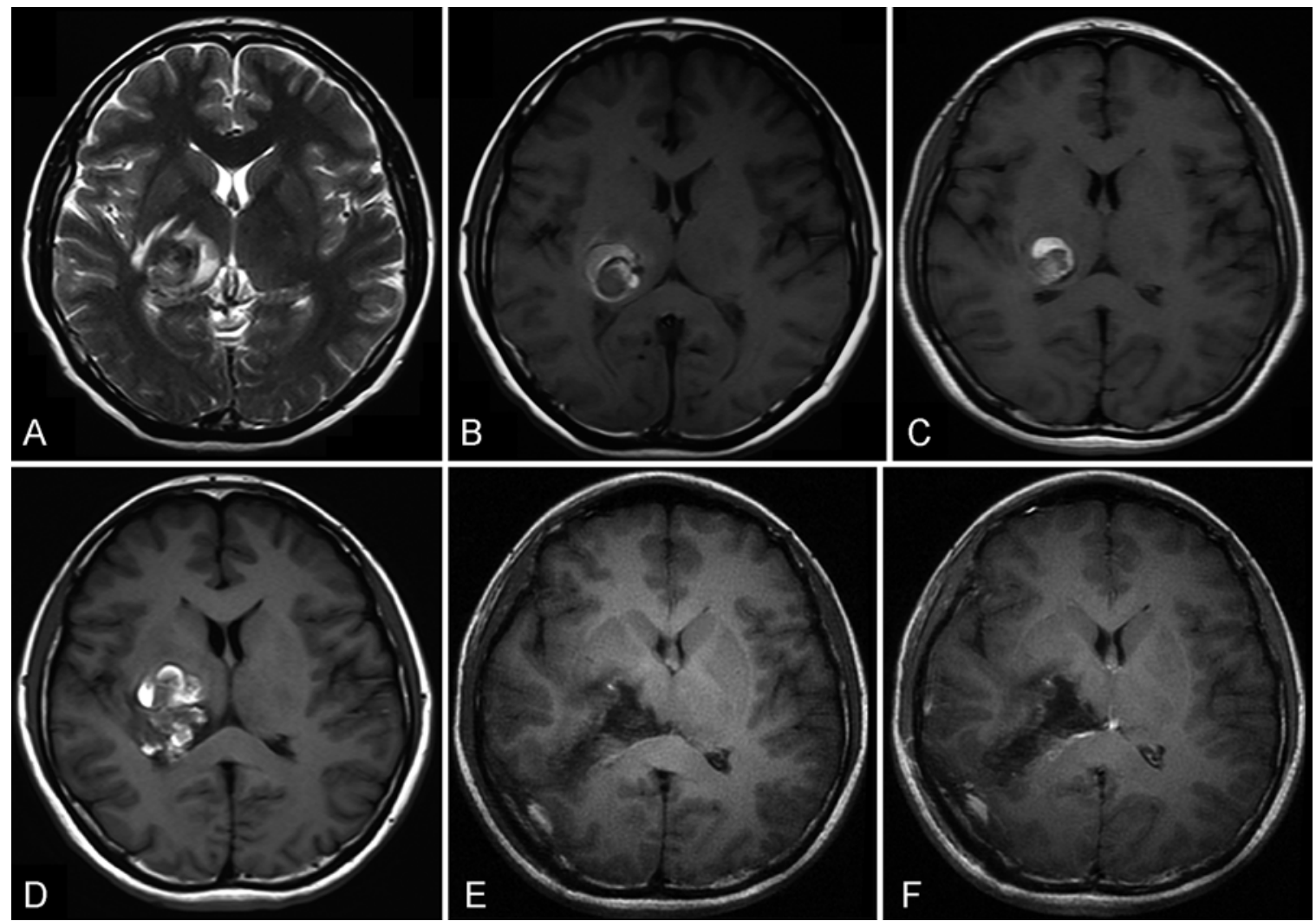

FIG. 4. Case 3. Axial MR images obtained in a patient who was treated with surgical excision of her CM. This 28-year-old woman suffered a hemorrhagic ictus of a right thalamic CM with a mRS score of 3 at initial diagnosis. The lesion presented with surrounding edema and subacute hemorrhage on T2-weighted (A) and T1-weighted (B) MRI. Additional hemorrhages occurred at 51.7 and 76.0 months after diagnosis and were identified based on T1-weighted images ( $\mathbf{C}$ and $\mathbf{D}$, respectively). Although the preoperative $\mathrm{mRS}$ score was 2, the patient underwent resection of the $\mathrm{CM}$ via a parietooccipital transventricular approach. The postoperative T1-weighted images obtained before $(\mathbf{E})$ and $\operatorname{after}(\mathbf{F})$ administration of a contrast agent indicated complete resection.

simple observation may no longer be sufficient for surgical candidates. Otherwise, patients without rehemorrhage or those who declined surgical treatment after rehemorrhage were simply followed with observation.

\section{Hemorrhage Rate}

From the time of diagnosis, 42 definite hemorrhages occurred in 35 patients ( 1 ictus in 28 patients and 2 in 7 patients). Of these 35 patients, 13 also had probable hemorrhages; the annual probable hemorrhage rate was estimated to be $3.5 \%$. There was no asymptomatic hemorrhage. The mortality rate for patients with definite hemorrhage was $8.6 \%$ (3 deaths among 35 patients). The remaining 86 patients $(71.7 \%)$ were free from hemorrhage after a mean follow-up duration of 3.1 years (range $0.1-16.6$ years). The annual definite hemorrhage rate was calculated to be $9.7 \%$ (Table 2). The rate was highest in patients initially presenting with hemorrhage and FND $(14.1 \%)\left(\chi^{2}=15.358, \mathrm{p}<\right.$ 0.001 ), followed by patients presenting with hemorrhage but without FND (4.5\%) and patients without hemorrhage regardless of symptoms (1.2\%). Other stratified annual hemorrhage rates are detailed in Table 3 and were significantly higher in patients with hemorrhagic presentation at diagnosis $(11.7 \%, p=0.004)$ or associated DVA $(15.7 \%, p$ $=0.002$ ).

The Kaplan-Meier actuarial overall hemorrhage-free survival rates for $1,2,5$, and 10 years were $90.1 \%, 77.9 \%$, $55.3 \%$, and $47.7 \%$, respectively (Fig. 5A). Irrespective of symptoms, the difference in hemorrhage-free survival between patients with or without an initial presentation of hemorrhage was significant $\left(\chi^{2}=6.935, \mathrm{p}=0.008\right)$ (Fig. $5 \mathrm{~B})$ according to Kaplan-Meier analysis. A multivariate Cox model was applied, and the initial presentation of hemorrhage with FND (hazard ratio [HR] 2.767, 95\% CI 1.336-5.731, $\mathrm{p}=0.006$ ) and associated DVA (HR 2.510, 95\% CI $1.275-4.942, \mathrm{p}=0.008$ ) were identified as independent hemorrhage risk factors that were illustrated by the Kaplan-Meier method (Fig. 5C and D). Other variables (i.e., age, sex, multiple CCMs, size, perilesional edema, depth of lesion, or Zabramski type) were not statistically significant factors.

\section{Neurological Outcomes of Untreated Thalamic CMs}

A follow-up duration of at least 1 year, at least 2 years, and at least 3 years recorded for 87 (71.9\%), 70 (57.9\%), and $57(47.1 \%)$ patients, respectively, and the mean mRS score at 1,2, and 3 years was $1.5,1.4$, and 1.3 , respectively. The mean mRS score at the last follow-up evaluation was 2.0 (range $0-6$ ) (Table 4), which was not significantly different from the mean mRS score at diagnosis $(\mathrm{t}$ $=1.378, \mathrm{p}=0.171)$. Forty-six patients $(38.0 \%)$ were able to carry out full- or part-time work or household duties, 
TABLE 2. Incidence of hemorrhage after initial presentation stratified by initial presentation

\begin{tabular}{|c|c|c|c|c|c|}
\hline \multirow[b]{2}{*}{ Variable } & \multirow[b]{2}{*}{ Overall } & \multicolumn{3}{|c|}{ Initial Presentation } & \multirow[b]{2}{*}{ p Value } \\
\hline & & Hemorrhage w/ FND & Hemorrhage w/o FND & No Hemorrhage & \\
\hline No. of hemorrhages & 42 & 37 & 4 & 1 & \\
\hline Pts w/ hemorrhage $(\%)$ & $35(28.9)$ & $30(34.5)$ & $4(21.1)$ & $1(6.7)$ & $0.064 \dagger$ \\
\hline Pts w/ multiple hemorrhages (\%) & $7(5.8)$ & $7(8.0)$ & 0 & 0 & $0.091 \dagger$ \\
\hline Mean follow-up duration, yrs \pm SD & $3.6 \pm 3.4$ & $3.0 \pm 3.0$ & $4.6 \pm 4.1$ & $5.5 \pm 4.2$ & $0.009^{*} \ddagger$ \\
\hline Median follow-up duration, yrs & 2.8 & 2.0 & 4.8 & 4.5 & \\
\hline Cumulative follow-up period, pt-yrs & 433.1 & 261.6 & 88.1 & 83.4 & \\
\hline Annual postpresentation hemorrhage rate, $\%$ & 9.7 & 14.1 & 4.5 & 1.2 & $<0.001^{*} \dagger$ \\
\hline
\end{tabular}

42 patients $(34.7 \%)$ were at least independent in daily life activity, and 30 patients (24.8\%) required assistance. Compared with mRS scores at diagnosis, recent mRS scores were improved in 37 patients (30.6\%), stable in 59 patients (48.8\%), and worsened in 25 patients (20.7\%). In patients with an initial presentation of hemorrhage and FND ( $\mathrm{n}=$ 87 ), the most recent mean mRS (score $2.3, p=0.002$ ) was significantly higher than in the other 2 subgroups.

Multivariate logistic regression analysis identified lesion size at diagnosis (per $0.1 \mathrm{~cm}$ increase) (odds ratio
[OR] 3.410, 95\% CI 1.272-9.146, $\mathrm{p}=0.015)$ and initial mRS score (per 1 point increase) (OR 3.548, 95\% CI 1.815-6.937, $\mathrm{p}<0.001)$ as independent adverse predictors for poor neurological outcome (mRS score $\geq 2$ ). However, prolonged posthemorrhagic period (per 0.1 month increase) improved the mRS score at follow-up (OR 0.932, 95\% CI $0.907-0.958, \mathrm{p}<0.001$ ). Other variables (i.e., age, sex, initial presentation, multiple CCMs, perilesional edema, DVA, depth of lesion, Zabramski type, or hemorrhage subsequent to initial presentation) were insignificant. Ad-

TABLE 3. Stratified annual hemorrhage rates for hemorrhage subsequent to initial presentation

\begin{tabular}{|c|c|c|c|c|c|}
\hline Subgroup & No. of Hemorrhages & Pt-Yrs & Annual Hemorrhage Rate, \% & $\chi^{2} \dagger$ & $\mathrm{p}$ Value \\
\hline All pts & 42 & 433.1 & 9.7 & & \\
\hline Sex & & & & 2.360 & 0.124 \\
\hline Female & 17 & 224.0 & 7.6 & & \\
\hline Male & 25 & 209.1 & 12.0 & & \\
\hline Hemorrhage at initial presentation & & & & 8.460 & $0.004^{*}$ \\
\hline Yes & 41 & 349.7 & 11.7 & & \\
\hline No & 1 & 83.4 & 1.2 & & \\
\hline Multiple hemorrhages & & & & 1.059 & 0.303 \\
\hline Yes & 8 & 111.0 & 7.2 & & \\
\hline No & 34 & 322.1 & 10.6 & & \\
\hline Edema & & & & 0.110 & 0.741 \\
\hline Yes & 21 & 227.0 & 9.3 & & \\
\hline No & 21 & 206.1 & 10.2 & & \\
\hline DVA & & & & 9.761 & $0.002^{*}$ \\
\hline Yes & 24 & 152.5 & 15.7 & & \\
\hline No & 18 & 280.6 & 6.4 & & \\
\hline Lesion depth & & & & 3.106 & 0.212 \\
\hline Deep & 5 & 89.5 & 5.6 & & \\
\hline Moderate & 9 & 105.7 & 8.5 & & \\
\hline Superficial & 28 & 237.9 & 11.8 & & \\
\hline Zabramski type & & & & 5.375 & 0.068 \\
\hline I & 13 & 126.2 & 10.3 & & \\
\hline II & 28 & 246.4 & 11.4 & & \\
\hline III/IV & 1 & 60.5 & 1.6 & & \\
\hline
\end{tabular}


A

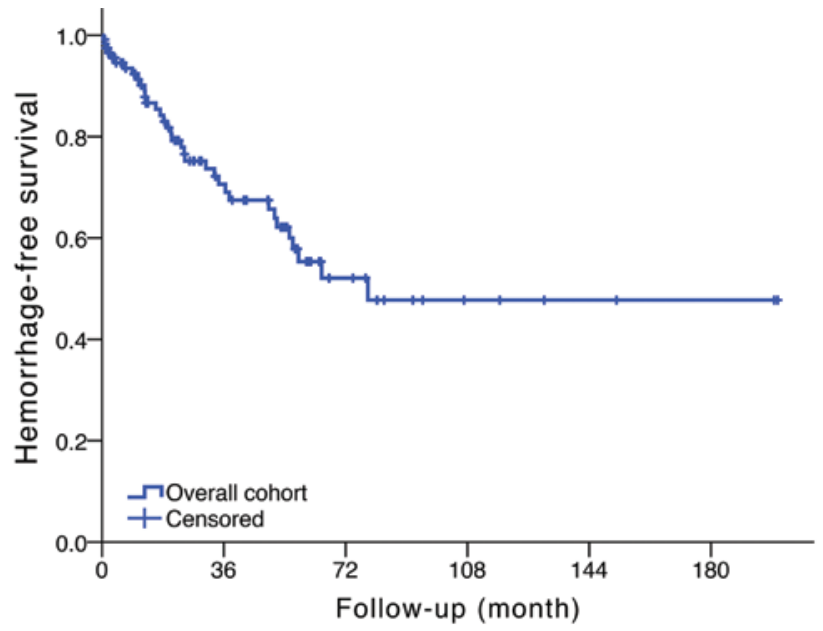

No. at risk

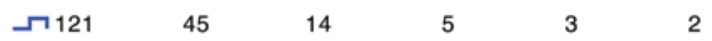

\section{C}

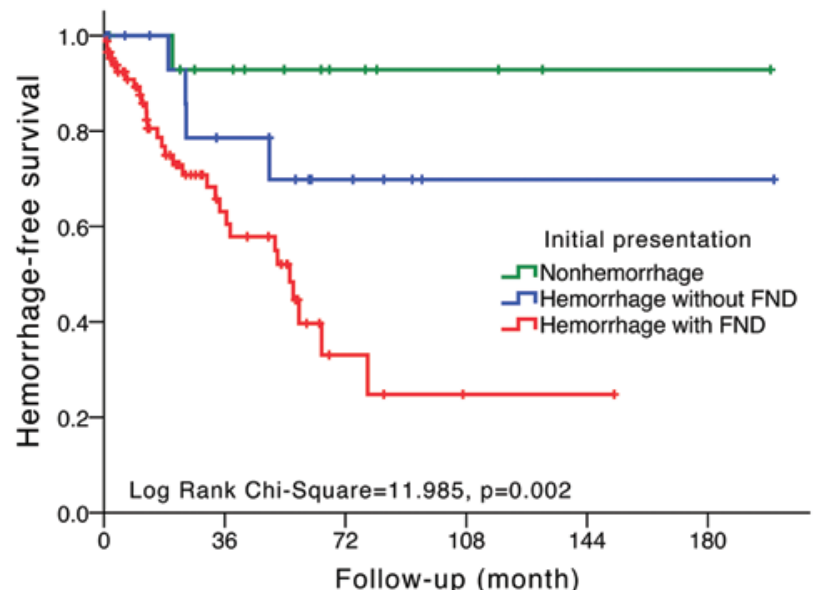

No. at risk

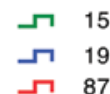

Follow-up (month)

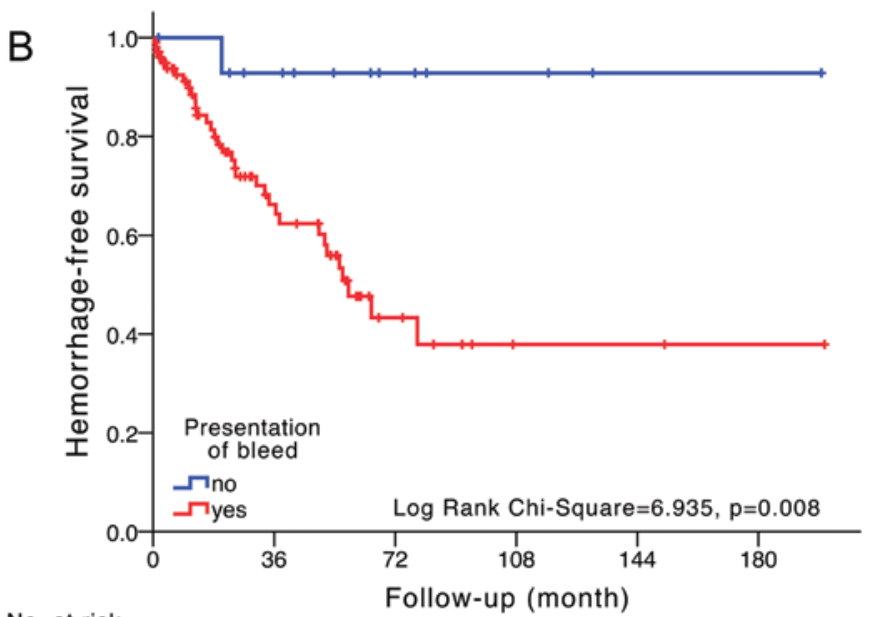

No. at risk

$\begin{array}{llllll}\neg 15 & 11 & 5 & 3 & 1 & 1 \\ \neg 106 & 34 & 9 & 2 & 2 & 1\end{array}$

$\mathrm{D}$

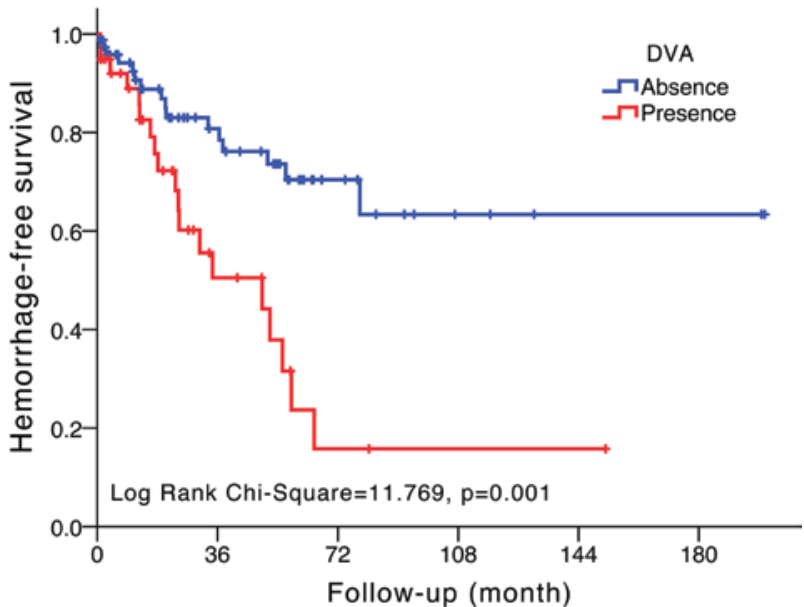

No. at risk

$\begin{array}{llllll}\sim 82 & 35 & 12 & 4 & 2 & 2 \\ \neg 39 & 10 & 2 & 1 & 1 & 0\end{array}$

FIG. 5. Kaplan-Meier analysis of hemorrhage-free survival. A: The estimated median hemorrhage-free survival time for the overall cohort was 78.6 months. B: Kaplan-Meier analysis showed a significant difference in hemorrhage-free survival between patients with or without an initial presentation of hemorrhage, and a presentation of hemorrhage predicted the trend of subsequent hemorrhage. C: Hemorrhage-free survival was worse for patients with an initial presentation of hemorrhage and FND than for patients without hemorrhagic presentation and for those with hemorrhage but without FND $(p=0.002)$. D: Patients with associated DVA were more likely to experience prospective hemorrhage than those without DVA $(p=0.001)$. Figure is available in color online only.

ditionally, patients experiencing hemorrhage (per 1 ictus increase) (OR 6.923, 95\% CI 3.023-15.855, p < 0.001) had a greater chance of a worse outcome.

\section{Discussion}

Thalamic CMs are relatively rare and have been reported to account for 5\%-17\% of CCMs; the mean rate was $8.2 \%$ across 7 previous studies dedicated to natural history. ${ }^{19}$ Hemorrhagic ictus or mass effect could cause conspicuous deterioration of neurological function due to the presumed hypersensitivity of essential tissues to even micromorphological changes. ${ }^{16,45}$ The risk of future hemorrhage has not been predictable, although it is critical in determining patient management strategy and prognosis.
In the present series, we found the following. 1) The annual definite hemorrhage rate of thalamic CMs was estimated to be $9.7 \%$, which significantly increased to $11.7 \%$ in patients with an initial presentation of hemorrhage and further increased to $15.7 \%$ in patients with associated DVA, and a relatively small percentage of patients (33.1\%) underwent resection. 2) Increased lesion size and high $\mathrm{mRS}$ score at diagnosis predicted poor outcome. 3) At the study end point, neurological function was improved/unchanged in $79.3 \%$ of patients, although this rate decreased to $45.7 \%$ $(16 / 35)$ in patients who had hemorrhages during the follow-up period (i.e., after the initial presentation). Based on these findings, we offer several observations. 1) The mode of initial presentation was useful for the prediction of hemorrhagic and neurological outcomes. 2) Patients with these 
TABLE 4. Functional outcome for patients with thalamic CMs classified by initial presentation

\begin{tabular}{|c|c|c|c|c|c|}
\hline Variable & Overall & Hemorrhage w/ FND & Hemorrhage w/o FND & No Hemorrhage & $\mathrm{p}$ Value \\
\hline \multicolumn{6}{|l|}{ Recent mRS score } \\
\hline Mean \pm SD & $2.0 \pm 1.3$ & $2.3 \pm 1.2$ & $1.4 \pm 1.1$ & $1.3 \pm 1.4$ & $0.002^{*} \dagger$ \\
\hline 0 & 11 & 3 & 3 & 5 & \\
\hline 1 & 35 & 20 & 9 & 6 & \\
\hline 2 & 42 & 36 & 5 & 1 & \\
\hline 3 & 15 & 14 & 0 & 1 & \\
\hline 4 & 15 & 11 & 2 & 2 & \\
\hline 6 & 3 & 3 & 0 & 0 & \\
\hline \multicolumn{6}{|l|}{ Clinical condition (\%) } \\
\hline Improved & $37(30.6)$ & $27(31.0)$ & $4(21.1)$ & $6(40.0)$ & $0.592 \ddagger$ \\
\hline Stable & $59(48.8)$ & $40(46.0)$ & $12(63.2)$ & $7(46.7)$ & \\
\hline Worsened & $25(20.7)$ & $20(23.0)$ & $3(15.8)$ & $2(13.3)$ & \\
\hline Censored due to GK (\%) & $15(12.4)$ & $9(10.3)$ & $4(21.1)$ & $2(13.3)$ & $0.436 \ddagger$ \\
\hline Censored due to surgery (\%) & $40(33.1)$ & $30(34.5)$ & $8(42.1)$ & $2(13.3)$ & $0.181 \ddagger$ \\
\hline
\end{tabular}

hemorrhagic risks required close follow-up and repeated radiological examinations whenever possible if a clinical ictus was suspected. 3) Given the low hemorrhagic mortality rate $(2.5 \%)$ and relatively acceptable overall rate of improved/unchanged condition, patient observation was adequate in the absence of a surgical indication or rejection of surgery. 4) Finally, hemorrhage during the followup period accompanied by clinical aggravation justified resection.

\section{Hemorrhage Rate}

The natural history of CCMs has been studied extensively. $5,11,13,15,18,19,21,23,26-31,38,39,45,50,52,53$ Thalamic CMs are rare, however, and usually reported together with basal ganglia CMs or classified as deep CMs, without specific clinical data for this subset. ${ }^{1}$ Previously, age, ${ }^{2,8,30}$ female $^{2,5,38,50}$ or male $^{15}$ sex, prior hemorrhage, $2,15,18,21-23,26,38,45$ symptomatic lesion, ${ }^{3}$ eloquent location, $, 11,18,21,29,45$ Zabramski classification (Type I/II), ${ }^{22}$ and DVA ${ }^{18}$ were associated with subsequent hemorrhage. However, results from various studies have been inconsistent. Some studies failed to identify the presence of deep CMs as a risk factor for hemorrhage. .,26,38 $^{2}$ In contrast, Cantu et al. ${ }^{11}$ and Porter et al. ${ }^{45}$ reported a significantly higher annual rate of hemorrhage for deep CMs than lobar/superficial CMs. The adverse factors identified in our study were similar to those described in previous studies, including hemorrhagic presentation, ${ }^{45}$ which strongly predicted future episodes of hemorrhage. Given the rupture possibility of DVAs and the intimate interaction between CMs and DVAs, CMs conferred an additional risk of extra- and intralesional hemorrhage, as illustrated in Case 2 (Fig. 3).

Gross et al. ${ }^{16}$ described annual hemorrhage rates ranging from $2.8 \%$ to $4.1 \%$, with elevated rehemorrhage rates of $6.1 \%$ to $11.0 \%{ }^{2}$ Similarly, an annual rate of CCMs reported by Gross et al..$^{18}$ was as low as $3.3 \%$ and increased to $11.3 \%$ with hemorrhagic presentation. The annual hemorrhage rate was $9.7 \%$ in our study, which was quite similar to the $11.0 \%$ for reported for basal ganglia CMs, ${ }^{2}$ comparable to rates of $13.6 \%{ }^{30}$ and $16.7 \%$ reported for brainstem CMs, and substantially lower than the $21.5 \%$ reported by Aiba et al. ${ }^{2}$ for brainstem CMs. Our annual rate was significantly lower than that reported in radiosurgical $(31.6 \%-32.0 \%)^{25,46}$ or surgical $(\sim 32.7 \%)$ studies. ${ }^{43}$ Lesions in noneloquent areas may go undetected without scheduled, repeated monitoring via radiological films. ${ }^{16}$ In the study by Zabramski et al. ${ }^{53}$ the hemorrhage rate doubled from $6.5 \%$ to $13 \%$ if asymptomatic or minimally symptomatic (i.e., headache) ictus was included. The high rate in the current study was attributed to a high percentage of patients with hemorrhagic presentation (87.6\%), prior hemorrhage (93.4\%), and DVA (32.2\%). Prior hemorrhage might imply that dilated sinusoidal vessels were less resistant to increases in blood pressure and to the inherent, potential trend of rupture after the "breakthrough of zero" (first hemorrhage) of an originally indolent, unruptured nidus. Another plausible explanation for the increased rate could be that the vital nucleus and fasciculus-intensive area of restricted space was susceptible to mass effect, ischemia, or damage caused by hematoma or thrombosis. Alternatively, some structural distinction or different deep venous drainage system could influence nidus rupture. ${ }^{45}$

\section{Neurological Outcomes}

Neurological function outcomes based on hemorrhage have not been extensively evaluated in any study that we were able to identify in the literature, including the metaanalysis of 11 studies describing 61 cases of basal ganglia or thalamic CMs. ${ }^{19}$ In 7 cases reported by Aiba et al. ${ }^{2}$ outcomes were described as excellent in 4 (57.1\%), but in the remaining 3 cases $(42.9 \%)$, the patients had moderate disabilities, with recurrent bleeds in the eloquent area being responsible for the disabilities. In the study by Porter et al., ${ }^{45}$ which focused on the natural history of cases of 
CCM in the absence of neurosurgical treatment, approximately $37 \%$ of patients with neurological deficits recovered fully, 36\% achieved some recovery, and $27 \%$ did not exhibit significant improvement in neurological status. The neurological morbidity observed in a study by Pozzati $^{46}$ of thalamic cavernous angiomas was $40 \%$. No fatalities related to hemorrhage were reported in a majority of studies, $2,3,15,18,19,22,29,31,38,53$ but the $4.3 \%$ mortality rate in the study by Al-Shahi Salman et al. ${ }^{5}$ was similar to the $8.6 \%$ rate in our study. In the current study, we compared outcomes based on the mode of initial presentation (Table 4), and as expected, favorable outcomes were more likely in patients without hemorrhage at diagnosis or without subsequent hemorrhagic ictus.

Multivariate analysis identified increased lesion size and high $\mathrm{mRS}$ score at diagnosis as independent risk factors for poor neurological outcome (mRS score $\geq 2$ ). An extended posthemorrhagic duration favored neurological recovery, and we postulated that regular rehabilitation and exercise would promote recovery during the event-free period rather than endorsing a theory of a time limit for recovery. ${ }^{45}$ If risk factors were undetermined at the time of diagnosis, a wait-and-see approach was employed. If large lesion size and poor mRS score were encountered, early surgical intervention was preferred to eliminate hemorrhage risk and to prevent neurological decline. ${ }^{16,33,48}$ Overall, outcome could be improved if surgery or observation was recommended to selected patients based on individual risk factors.

\section{Implications for Management Strategy}

The hemorrhage rate stratified by characteristics present in the initial presentation can be compared with the risks associated with surgical or radiosurgical intervention to determine an appropriate treatment course. According to the surgical guidelines at our institution, only a few patients $(18.9 \%$, Fig. 1) were referred to surgery immediately (but not on an emergency basis) after diagnosis, and the percentage of patients requiring surgical intervention during observation was $33.1 \%$. Both of these rates were significantly inflated due to tertiary referral bias. Given the theory that early postoperative outcome might mimic an additional hemorrhagic event, ${ }^{17}$ we roughly compared the surgical outcomes reported in 4 prior studies ${ }^{16,33,43,48}$ with the present study and found that the previously reported improved/stabilized rates of $91.3 \%,{ }^{48} 92.6 \%, 3380.0 \%, 43$ and $75.0 \% 16,33$ fell in between those of patients without subsequent hemorrhagic ictus $(93.0 \%, 80 / 86)$ and those with subsequent hemorrhagic ictus $(45.7 \%)$. These findings supported the strategy of delayed surgery, which was reserved for patients who did not have surgical indications at initial diagnosis but subsequently suffered a second hemorrhage. The prospect of a future hemorrhage shifted patients' attitudes toward surgical intervention from hesitant to much more positive. Detailed consultation with patients and family members was necessary to thoroughly communicate the risks and benefits and, most importantly, to allow them to participate in treatment decisions. Although the validity of the comparison was compromised by the heterogeneity in these studies, no available literature was found that could address this issue; prospective randomized controlled trials are needed.
The present study did not aim to identify the superiority of observation over surgery. For most patients, a conservative attitude of wait and see was justified. Careful consideration was necessary before recommending surgery to patients with lesions that were particularly difficult to access, even when they had suffered multiple hemorrhages, because destruction of the normal parenchyma surrounding the lesion would likely lead to new deficits that could offset the benefits of surgery itself.

\section{Limitations of the Present Study}

Our follow-up methods constituted a limitation of our study, with assessment being performed at times via telephone or email, which made outcomes such as mRS and assessment of neurological events less valid. The fact that our patients came from across our country, not merely from regions surrounding our institution, reduced the percentage of patients voluntarily returning for clinical follow-up, especially for patients without any hemorrhagic ictus after their initial diagnosis. To improve the validity and accuracy of the neurological evaluation via telephone/ email and to minimize the bias caused by follow-up methods, evaluations were carefully performed according to a case report form, and in a few cases, photographs and/or a short video were obtained via email and were used in evaluations.

Initially, we excluded patients from natural history analysis if they had a censoring event within 12 months of diagnosis. Our reasons were as follows. 1) A duration of follow-up less than 12 months was not long enough compared with a patient's typical life expectancy to define the prognosis of the untreated lesion. 2) These subgroup patients had an inconspicuous effect on the overall annual hemorrhage rate, and they contributed only a small amount of information to the natural history study. 3) Tertiary referral bias resulted in a higher percentage of patients with severe lesions than one would see in the general population, and the cutoff of 12 months could minimize the bias in a sense. 4) The patients who underwent surgical treatment within 12 months were likely to have had lesions that were particularly aggressive rather than being representative of the whole spectrum of brainstem CMs (including relatively indolent lesions). To minimize the introduction of bias, we included patients with follow-up $<12$ months in the natural history analysis but that compromised the mean follow-up duration.

The tertiary care referrals in our institution presented an obvious recruiting bias compared with other recruitment criteria such as radiological screening ${ }^{3,15}$ and the population-based cohort study design..$^{5}$ This referral bias limited the generalizability of our findings and overestimated the annual hemorrhage rate of thalamic CMs. Our data were not derived from a wide variety of thalamic CMs but from a subset of predominantly aggressive CMs that included a very low percentage of quiescent lesions. This subset was responsible for the continual hemorrhagic ictus. Although asymptomatic/incidental lesions were underrepresented in the present study, our findings could be applicable in other tertiary referral centers and could provide specific extrapolation to a patient population with hemorrhagic, symptomatic CMs involving eloquent areas. ${ }^{19}$ A multicenter (at 
all levels) or population-based study should be considered in the future to minimize these biases.

\section{Conclusions}

This study verified adverse predictors for hemorrhage and functional outcomes of thalamic CMs and demonstrated an overall annual symptomatic hemorrhage rate of $9.7 \%$ after initial diagnosis. Clinical consultation should clearly indicate risks and benefits, and patients should be allowed to contribute to the treatment decision. The findings presented herein will be useful for clinicians and patients in the selection of an appropriate treatment plan. Our study was subject to referral bias due to the tertiary care setting, and multicenter prospective studies are needed to validate our findings.

\section{Acknowledgments}

This study was supported by the Center of Brain Tumor, Beijing Institute for Brain Disorders (Grant No. BIBDPXM2013_014226_07_000084), and the National Key Technology Research and Development Program of the Ministry of Science and Technology of China (Grant No. 2013BAI09B03).

\section{References}

1. Abla AA, Spetzler RF: Cavernous malformations of the thalamus: a relatively rare but controversial entity. World Neurosurg 79:641-644, 2013

2. Aiba T, Tanaka R, Koike T, Kameyama S, Takeda N, Komata T: Natural history of intracranial cavernous malformations. J Neurosurg 83:56-59, 1995

3. Al-Holou WN, O’Lynnger TM, Pandey AS, Gemmete JJ, Thompson BG, Muraszko KM, et al: Natural history and imaging prevalence of cavernous malformations in children and young adults. J Neurosurg Pediatr 9:198-205, 2012

4. Al-Shahi Salman R, Berg MJ, Morrison L, Awad IA: Hemorrhage from cavernous malformations of the brain: definition and reporting standards. Stroke 39:3222-3230, 2008

5. Al-Shahi Salman R, Hall JM, Horne MA, Moultrie F, Josephson CB, Bhattacharya JJ, et al: Untreated clinical course of cerebral cavernous malformations: a prospective, populationbased cohort study. Lancet Neurol 11:217-224, 2012

6. Al-Shahi Salman R, Kitchen N, Thomson J, Ganesan V, Mallucci C, Radatz M: Top ten research priorities for brain and spine cavernous malformations. Lancet Neurol 15:354-355, 2016

7. Banks JL, Marotta CA: Outcomes validity and reliability of the modified Rankin scale: implications for stroke clinical trials: a literature review and synthesis. Stroke 38:10911096, 2007

8. Barker FG II, Amin-Hanjani S, Butler WE, Lyons S, Ojemann RG, Chapman PH, et al: Temporal clustering of hemorrhages from untreated cavernous malformations of the central nervous system. Neurosurgery 49:15-25, 2001

9. Bertalanffy H, Benes L, Miyazawa T, Alberti O, Siegel AM, Sure U: Cerebral cavernomas in the adult. Review of the literature and analysis of 72 surgically treated patients. Neurosurg Rev 25:1-55, 2002

10. Cakirer S: De novo formation of a cavernous malformation of the brain in the presence of a developmental venous anomaly. Clin Radiol 58:251-256, 2003

11. Cantu C, Murillo-Bonilla L, Arauz A, Higuera J, Padilla J, Barinagarrementeria F: Predictive factors for intracerebral hemorrhage in patients with cavernous angiomas. Neurol Res 27:314-318, 2005
12. Chang EF, Gabriel RA, Potts MB, Berger MS, Lawton MT: Supratentorial cavernous malformations in eloquent and deep locations: surgical approaches and outcomes. Clinical article. J Neurosurg 114:814-827, 2011

13. Del Curling O Jr, Kelly DL Jr, Elster AD, Craven TE: An analysis of the natural history of cavernous angiomas. J Neurosurg 75:702-708, 1991

14. Ferroli P, Sinisi M, Franzini A, Giombini S, Solero CL, Broggi G: Brainstem cavernomas: long-term results of microsurgical resection in 52 patients. Neurosurgery 56:12031214,2005

15. Flemming KD, Link MJ, Christianson TJ, Brown RD Jr: Prospective hemorrhage risk of intracerebral cavernous malformations. Neurology 78:632-636, 2012

16. Gross BA, Batjer HH, Awad IA, Bendok BR: Cavernous malformations of the basal ganglia and thalamus. Neurosurgery 65:7-19, 2009

17. Gross BA, Batjer HH, Awad IA, Bendok BR, Du R: Brainstem cavernous malformations: 1390 surgical cases from the literature. World Neurosurg 80:89-93, 2013

18. Gross BA, Du R, Orbach DB, Scott RM, Smith ER: The natural history of cerebral cavernous malformations in children. J Neurosurg Pediatr 17:123-128, 2016

19. Gross BA, Lin N, Du R, Day AL: The natural history of intracranial cavernous malformations. Neurosurg Focus 30(6):E24, 2011

20. Henrich W, Stupin JH, Bühling KJ, Bührer C, Bassir C, Dudenhausen JW: Prenatal sonographic findings of thalamic cavernous angioma. Ultrasound Obstet Gynecol 19:518522,2002

21. Horne MA, Flemming KD, Su IC, Stapf C, Jeon JP, Li D, et al: Clinical course of untreated cerebral cavernous malformations: a meta-analysis of individual patient data. Lancet Neurol [epub ahead of print], 2015

22. Jeon JS, Kim JE, Chung YS, Oh S, Ahn JH, Cho WS, et al: A risk factor analysis of prospective symptomatic haemorrhage in adult patients with cerebral cavernous malformation. J Neurol Neurosurg Psychiatry 85:1366-1370, 2014

23. Kim DS, Park YG, Choi JU, Chung SS, Lee KC: An analysis of the natural history of cavernous malformations. Surg Neurol 48:9-18, 1997

24. Kobata H, Kondo A, Iwasaki K, Hattori I: Massive subependymal hemorrhage caused by an occult vascular malformation - two case reports. Neurol Med Chir (Tokyo) 39:302307, 1999

25. Kondziolka D, Lunsford LD, Flickinger JC, Kestle JR: Reduction of hemorrhage risk after stereotactic radiosurgery for cavernous malformations. J Neurosurg 83:825-831, 1995

26. Kondziolka D, Lunsford LD, Kestle JR: The natural history of cerebral cavernous malformations. J Neurosurg 83:820824,1995

27. Kupersmith MJ, Kalish H, Epstein F, Yu G, Berenstein A, Woo H, et al: Natural history of brainstem cavernous malformations. Neurosurgery 48:47-54, 2001

28. Labauge P, Brunereau L, Laberge S, Houtteville JP: Prospective follow-up of 33 asymptomatic patients with familial cerebral cavernous malformations. Neurology 57:1825-1828, 2001

29. Labauge P, Brunereau L, Lévy C, Laberge S, Houtteville JP: The natural history of familial cerebral cavernomas: a retrospective MRI study of 40 patients. Neuroradiology 42:327-332, 2000

30. Li D, Hao SY, Jia GJ, Wu Z, Zhang LW, Zhang JT: Hemorrhage risks and functional outcomes of untreated brainstem cavernous malformations. J Neurosurg 121:32-41, 2014

31. Li D, Hao SY, Tang J, Xiao XR, Jia GJ, Wu Z, et al: Clinical course of untreated pediatric brainstem cavernous malformations: hemorrhage risk and functional recovery. J Neurosurg Pediatr 13:471-483, 2014 
32. Li D, Yang Y, Hao SY, Wang L, Tang J, Xiao XR, et al: Hemorrhage risk, surgical management, and functional outcome of brainstem cavernous malformations. J Neurosurg 119:996-1008, 2013

33. Li D, Zhang J, Hao S, Tang J, Xiao X, Wu Z, et al: Surgical treatment and long-term outcomes of thalamic cavernous malformations. World Neurosurg 79:704-713, 2013

34. Lin F, Wu J, Wang L, Zhao B, Tong X, Jin Z, et al: Surgical treatment of cavernous malformations involving the posterior limb of the internal capsule: utility and predictive value of preoperative diffusion tensor imaging. World Neurosurg 88:538-547, 2016

35. Mathiesen T, Edner G, Kihlström L: Deep and brainstem cavernomas: a consecutive 8-year series. J Neurosurg 99:3137, 2003

36. Mehdorn HM, Barth H, Buhl R, Nabavi A, Weinert D: Intracranial cavernomas: indications for and results of surgery. Neurol Med Chir (Tokyo) 38 Suppl:245-249, 1998

37. Mhoyan A, Fulbright R, Bannykh SI: Cavernous hemangioma of the thalamus. J Neurooncol 71:257, 2005

38. Moriarity JL, Wetzel M, Clatterbuck RE, Javedan S, Sheppard JM, Hoenig-Rigamonti K, et al: The natural history of cavernous malformations: a prospective study of 68 patients. Neurosurgery 44:1166-1173, 1999

39. Nikoubashman O, Di Rocco F, Davagnanam I, Mankad K, Zerah M, Wiesmann M: Prospective hemorrhage rates of cerebral cavernous malformations in children and adolescents based on MRI appearance. AJNR Am J Neuroradiol 36:2177-2183, 2015

40. Notelet L, Chapon F, Khoury S, Vahedi K, Chodkiewicz JP, Courtheoux P, et al: Familial cavernous malformations in a large French kindred: mapping of the gene to the CCM1 locus on chromosome 7q. J Neurol Neurosurg Psychiatry 63:40-45, 1997

41. Nyáry I, Major O, Hanzély Z, Szeifert GT: Histopathological findings in a surgically resected thalamic cavernous hemangioma 1 year after 40-Gy irradiation. J Neurosurg 102 Suppl:56-58, 2005

42. Otani N, Fujioka M, Oracioglu B, Muroi C, Khan N, Roth P, et al: Thalamic cavernous angioma: paraculminar supracerebellar infratentorial transtentorial approach for the safe and complete surgical removal. Acta Neurochir Suppl 103:29_ 36, 2008

43. Pandey P, Westbroek EM, Gooderham PA, Steinberg GK: Cavernous malformation of brainstem, thalamus, and basal ganglia: a series of 176 patients. Neurosurgery 72:573-589, 2013

44. Pannek HW, Oppel F: Minimally invasive lesionectomies through a stereotactically guided working sleeve. Neurol Res 21:51-59, 1999

45. Porter PJ, Willinsky RA, Harper W, Wallace MC: Cerebral cavernous malformations: natural history and prognosis after clinical deterioration with or without hemorrhage. J Neurosurg 87:190-197, 1997
46. Pozzati E: Thalamic cavernous malformations. Surg Neurol 53:30-40, 2000

47. Pozzati E, Acciarri N, Tognetti F, Marliani F, Giangaspero F: Growth, subsequent bleeding, and de novo appearance of cerebral cavernous angiomas. Neurosurgery 38:662-670, 1996

48. Rangel-Castilla L, Spetzler RF: The 6 thalamic regions: surgical approaches to thalamic cavernous malformations, operative results, and clinical outcomes. J Neurosurg 123:676-685, 2015

49. Rigamonti D, Drayer BP, Johnson PC, Hadley MN, Zabramski J, Spetzler RF: The MRI appearance of cavernous malformations (angiomas). J Neurosurg 67:518-524, 1987

50. Robinson JR, Awad IA, Little JR: Natural history of the cavernous angioma. J Neurosurg 75:709-714, 1991

51. Tew JM Jr, Lewis AI, Reichert KW: Management strategies and surgical techniques for deep-seated supratentorial arteriovenous malformations. Neurosurgery 36:1065-1072, 1995

52. Washington CW, McCoy KE, Zipfel GJ: Update on the natural history of cavernous malformations and factors predicting aggressive clinical presentation. Neurosurg Focus 29(3):E7, 2010

53. Zabramski JM, Wascher TM, Spetzler RF, Johnson B, Golfinos J, Drayer BP, et al: The natural history of familial cavernous malformations: results of an ongoing study. J Neurosurg 80:422-432, 1994

\section{Disclosures}

The authors report no conflict of interest concerning the materials or methods used in this study or the findings specified in this paper.

\section{Author Contributions}

Conception and design: all authors. Acquisition of data: Li, Tian, Zheng, Ma, Wang. Analysis and interpretation of data: all authors. Drafting the article: Li, Tian, Zheng, Ma, Wu. Critically revising the article: all authors. Reviewed submitted version of manuscript: all authors. Approved the final version of the manuscript on behalf of all authors: Li. Statistical analysis: Li, Tian, Zheng, Ma, Wang, Wu. Administrative/technical/material support: Li, Tian, Zheng, Ma, Hao, LW Zhang, JT Zhang. Study supervision: all authors.

\section{Correspondence}

Da Li, Department of Neurosurgery, Beijing Tiantan Hospital, Capital Medical University, Tiantan Xili 6, Dongcheng District, Beijing 100050, People's Republic of China. email: lidaatlas@ aliyun.com. 